\title{
To: Mathieu C, Gysemans C, Giulietti A, Bouillon R (2005) Vitamin D and diabetes. Diabetologia 48:1247-1257
}

Received: 24 August 2005 / Accepted: 2 September 2005 / Published online: 13 December 2005

(C) Springer-Verlag 2005

To the Editor: Recently, an insightful review by Mathieu and co-workers on the role of vitamin D in the development of diabetes was published [1]. We would like to comment on the interpretation of the evidence for a relation between vitamin $\mathrm{D}$ status and type 2 diabetes in humans, and also present new data on this topic.

First, the results in humans have been more varied than is suggested in the review and may not warrant the conclusion that 'solid evidence exists that vitamin D deficiency leads to glucose intolerance in humans'. The authors did not refer to several important, larger studies in this area [2-7] that did not all consistently show an association between vitamin D status and type 2 diabetes or related parameters [4-6].

Second, in addition to the mechanisms described by Mathieu et al., it has been previously suggested that the association between vitamin $\mathrm{D}$ and glucose tolerance may be explained by parathyroid hormone (PTH). Serum 1,25dihydroxy-vitamin $\mathrm{D}\left(1,25-(\mathrm{OH})_{2}-\mathrm{D}\right)$ is required for normal insulin secretion, possibly through an effect of PTH on intra-cellular free calcium concentrations of the pancreatic beta cell [8]. In addition, PTH may influence insulin sensitivity by intra-cellular calcium changes in adipocytes and

M. Snijder · R. van Dam · M. Visser · J. Seidell

Institute for Health Sciences,

Free University of Amsterdam,

Amsterdam, The Netherlands

M. Snijder $(\bowtie) \cdot$ R. van Dam $\cdot$ M. Visser .

D. Deeg · J. Seidell · P. Lips

Institute for Research in Extramural Medicine,

VU University Medical Center,

Van der Boechorststraat 7,

1081 BT Amsterdam, The Netherlands

e-mail: marieke.snijder@falw.vu.nl

Tel.: +31-20-4449946

Fax: $+31-20-4446940$

P. Lips

Department of Endocrinology,

VU University Medical Center,

Amsterdam, The Netherlands muscle cells [9]. Wareham et al. have shown that PTH concentrations were independently associated with glucose tolerance, whereas vitamin D status was not [4].

Therefore, we examined the association of serum 25hydroxy-vitamin D (25-OH-D) and PTH concentrations with type 2 diabetes in a large study of older men and women, who are highly susceptible to vitamin D deficiency and type 2 diabetes. In 1,235 participants $(49.7 \%$ men, mean age $75.4 \pm 6.5$ years) of the Longitudinal Aging Study Amsterdam (1995/96 examination), serum 25-OH-D was determined using a competitive protein binding assay (Nichols Diagnostics, San Juan Capistrano, CA, USA) and PTH levels were determined by means of immunoradiometric assay (Incstar, Stillwater, MN, USA). The presence of diabetes was assessed with a detailed questionnaire on self-reported chronic diseases in combination with information obtained from the general practitioner. In addition, possible confounders were studied. Information on lifestyle factors (physical activity, alcohol intake and smoking) was obtained by questionnaires and anthropometric measurements (BMI, waist circumference) were performed. Also season, region of data collection (west, northeast, and south of the Netherlands), age, and sex were considered as potential confounders. Logistic regression analyses were performed to study the associations between 25-OH-D or PTH levels (independent variables) and the presence of type 2 diabetes (dependent variable). 25-OH-D was categorised into four groups based on proposed cut points [10], and the highest group ( $\geq 75 \mathrm{nmol} / \mathrm{l})$ was the reference group. PTH was categorised in tertiles and the lowest tertile was the reference group. We adjusted for potential confounders by adding these as independent variables to the regression models.

A vitamin-D-deficient status (25-OH-D levels $<25 \mathrm{nmol} / \mathrm{l}$ ) was found in 131 subjects (10.6\%), and 450 subjects (36.4\%) had insufficient vitamin D levels $(25-50 \mathrm{nmol} / \mathrm{l})$. In our sample, $108(8.7 \%)$ subjects (47 men and 61 women) had type 2 diabetes. The results of the logistic regression analyses are shown in Table 1 . There were no significant associations between 25-OH-D or PTH and the risk of having diabetes. 
Table 1 Risk (odds ratios with 95\% CIs) of having type 2 diabetes in categories of vitamin D (25-OH-D) and parathyroid hormone (PTH) concentrations

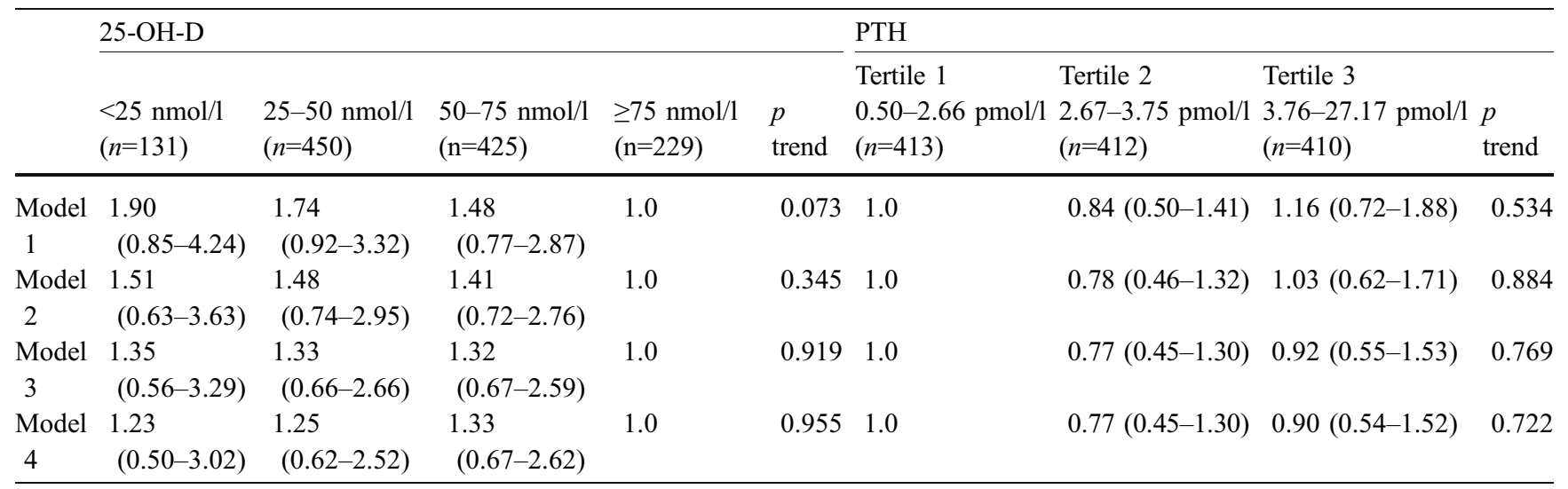

Model 1: crude model

Model 2: model 1 adjusted for age (years), sex, region and season

Model 3: model 2 additionally adjusted for waist circumference $(\mathrm{cm})$

Model 4: model 3 additionally adjusted for physical activity (min/day), alcohol intake, and smoking

$p$ for trend: by logistic regression analyses using 25-OH-D or PTH as continuous determinant of the presence of type 2 diabetes

The inconsistency between studies in results on vitamin D status and glucose tolerance may be caused by the characteristics of the study population, such as vitamin D status or ethnicity. Scragg et al. for example have shown that there was an independent association between 25-OH-D and risk of diabetes in non-Hispanic whites and Mexican-Americans, but no such association existed in non-Hispanic blacks [6]. Other studies including different ethnic groups did not report results of separate analyses for these groups $[2,5]$. It is possible that $25-\mathrm{OH}-\mathrm{D}$ only influences clinical type 2 diabetes in the deficient range of $25-\mathrm{OH}-\mathrm{D}$, which is more prevalent in some ethnic populations. Boucher et al. for example found that vitamin D deficiency was much more prevalent and severe in Asians living in East London than in older Caucasian people [11].

As argued in the review of Mathieu et al., the link between vitamin D status and glucose metabolism is of great interest and potentially important for public health. However, more extensive studies on vitamin D status and type 2 diabetes are needed to elucidate reasons for the varied results of studies in humans and to draw definite conclusions.

\section{References}

1. Mathieu C, Gysemans C, Giulietti A, Bouillon R (2005) Vitamin D and diabetes. Diabetologia 48:1247-1257

2. Scragg R, Holdaway I, Singh V, Metcalf P, Baker J, Dryson E (1995) Serum 25-hydroxyvitamin D3 levels decreased in impaired glucose tolerance and diabetes mellitus. Diabetes Res Clin Pract 27:181-188
3. Baynes KC, Boucher BJ, Feskens EJ, Kromhout D (1997) Vitamin D, glucose tolerance and insulinaemia in elderly men. Diabetologia 40:344-347

4. Wareham NJ, Byrne CD, Carr C, Day NE, Boucher BJ, Hales CN (1997) Glucose intolerance is associated with altered calcium homeostasis: a possible link between increased serum calcium concentration and cardiovascular disease mortality. Metabolism 46:1171-1177

5. Chiu KC, Chu A, Go VL, Saad MF (2004) Hypovitaminosis D is associated with insulin resistance and beta cell dysfunction. Am J Clin Nutr 79:820-825

6. Scragg R, Sowers M, Bell C (2004) Serum 25-hydroxyvitamin $\mathrm{D}$, diabetes, and ethnicity in the third national health and nutrition examination survey. Diabetes Care 27:2813-2818

7. Need AG, O'Loughlin PD, Horowitz M, Nordin BE (2005) Relationship between fasting serum glucose, age, body mass index and serum 25 hydroxyvitamin $\mathrm{D}$ in postmenopausal women. Clin Endocrinol (Oxf) 62:738-741

8. Lee S, Clark SA, Gill RK, Christakos S (1994) 1,25Dihydroxyvitamin D3 and pancreatic beta-cell function: vitamin $\mathrm{D}$ receptors, gene expression, and insulin secretion. Endocrinology 134:1602-1610

9. McCarty MF, Thomas CA (2003) PTH excess may promote weight gain by impeding catecholamine-induced lipolysisimplications for the impact of calcium, vitamin D, and alcohol on body weight. Med Hypotheses 61:535-542

10. Lips P (2001) Vitamin D deficiency and secondary hyperparathyroidism in the elderly: consequences for bone loss and fractures and therapeutic implications. Endocr Rev 22:477-501

11. Boucher BJ, Mannan N, Noonan K, Hales CN, Evans SJ (1995) Glucose intolerance and impairment of insulin secretion in relation to vitamin D deficiency in east London Asians. Diabetologia 38:1239-1245 